\title{
Risk factors for radiation pneumonitis caused by whole breast irradiation following breast-conserving surgery
}

\author{
Akiko Kubo', Kyosuke Osaki', Takashi Kawanaka', Shunsuke Furutani', \\ Hitoshi Ikushima ${ }^{2}$, and Hiromu Nishitani ${ }^{1}$ \\ ${ }^{1}$ Department of Radiology and ${ }^{2}$ Department of Radiation Therapy Technology, Institute of Health \\ Biosciences, the University of Tokushima Graduate School, Tokushima, Japan
}

\begin{abstract}
We evaluated risk factors of radiation pneumonitis (RP) after whole breast irradiation following breast-conserving surgery. Four hundred and seventy-two cases underwent whole breast irradiation with tangential field following breast-conserving surgery in our hospital, between January 2005 and April 2007. Of these cases, we performed statistical analyses for 423 breasts of 413 patients, using a pulmonary dose-volume histogram. Patient characteristics, treatment regimens and irradiation methods were included as variables in the analyses on risk factors of RP. As a result, 89 breasts of 84 cases (21\%) were diagnosed with RP. The version 3.0 of the NCI Common Terminology Criteria for Adverse Events was used to evaluate the grade of pneumonitis : 77 cases (18.2\%) were diagnosed as Grade $1 \mathrm{RP}, 10$ cases (2.3\%) as Grade 2, and 2 cases (0.5\%) as Grade 3. Multivariate analysis indicated that the significant risk factors for $R P$ were central lung distance (CLD) $(>1.8 \mathrm{~cm})$ and the short axis length of the radiation field. The incidence of radiationinduced bronchiolitis obliterans organizing pneumonia (BOOP) syndrome significantly correlated only with CLD. The lung volume within the radiation field was shown to be a significant risk factor for $\mathrm{RP}$ and radiation-induced BOOP syndrome. J. Med. Invest. 56 : 99-110, August, 2009
\end{abstract}

Keywords : radiation pneumonitis, radiation-induced BOOP syndrome, breast cancer, breast-conserving therapy

\section{INTRODUCTION}

Breast-conserving therapy (BCT) has recently become a standard treatment for early breast cancer. The local recurrence rate is decreased using postoperative radiotherapy, which cures minute lesions remaining within the breast (1). In recent years, an increasing number of patients have received breastconserving surgery. Even locally advanced cancers have received this treatment, following down-staging

Received for publication December 25, 2008 ; accepted January 26, 2009.

Address correspondence and reprint requests to Akiko Kubo, MD, Department of Radiology, Institute of Health Biosciences, the University of Tokushima Graduate School, Kuramoto-cho, Tokushima 770-8503, Japan and Fax +81-88-633-7174. with neo-adjuvant chemotherapy. The reported frequency of radiation pneumonitis (RP), caused by radiotherapy following breast-conserving surgery, varies greatly ; cumulative incidence rates of $0.9-85 \%$ have been reported (2-9). RP occurs locally, in a limited field of an irradiated lung, at an early time after irradiation(10). Among the pulmonary injuries following radiotherapy of whole breast, the most clinical significant pulmonary disorder is radiationinduced bronchiolitis obliterans organizing pneumonia (BOOP) syndrome. Radiation-induced BOOP syndrome is characterized by infiltrative shadow expansions outside the irradiation field of lung and migrates ; however, the frequency of radiation-induced BOOP is low. Crestani described the following factors for diagnosing radiation-induced BOOP 
syndrome (11) : (1) radiotherapy to the breast within the past 12 months, (2) general and/or respiratory symptoms lasting for longer than 2 weeks, (3) radiographic lung infiltrations outside the radiation port and (4) no evidence of a specific cause.

Previous clinical studies investigating RP have reported on several factors involved in the pathogenesis of RP, including patient age, lung volume within an irradiated field, chemotherapy before radiotherapy, prescription of tamoxifen during radiotherapy and smoking history (12-15). In the present study, we evaluated the risk factor for RP, including radiation-induced BOOP syndrome, induced by whole breast irradiation following breast-conserving surgery, with respect to patient background factors and treatment regimens.

\section{MATERIALS AND METHODS}

Between January 2005 and April 2007, 472 patient cases were subjected to tangential irradiation following breast-conserving surgery at the Tokushima University Hospital. We conducted follow-up observations for 454 of 472 patients with respect to the occurrence of pulmonary damage using chest radiographs. We excluded cases in which the chest wall had been irradiated after mastectomy, and in which irradiation on axilla and supraclavicular nodal regions had been simultaneously performed. We included only those cases in which whole breast tangential irradiation had been performed. In these cases, we performed statistical analyses for 423 breasts (413 cases) using a pulmonary dose-volume histogram (DVH). DVH was calculated using a three-dimensional treatment planning device (XiO, ver. 4.33.02, manufactured by CMS). Patient characteristics, treatment regimen and irradiation methods were included as variables on the analyses of the risk factors for RP. In 10 cases, tangential irradiation was performed simultaneously for both breasts following breast-conserving surgery.

\section{Patients}

The patient background of 413 cases is shown in Tables 1 and 2 . With respect to chemotherapy, neo-adjuvant chemotherapy was performed in 49 cases, pre-irradiation adjuvant chemotherapy in 5 cases and post-irradiation adjuvant chemotherapy in 100 cases. Of the 313 cases in which endocrine therapy was conducted, 130 concurrently received both endocrine therapy and radiotherapy, whereas
Table 1. Patient characteristics $(n=413)$

\begin{tabular}{|c|c|}
\hline Age (y), range (median) & $24-84(52)$ \\
\hline Side (right/left/bilateral) & $212 / 191 / 10$ \\
\hline Collagen vascular disease (yes/no) & $6 / 407$ \\
\hline Allergy disease and /or drug allergy (yes/no) & $73 / 340$ \\
\hline Diabetes (yes/no) & $12 / 401$ \\
\hline Smoking status (yes/no/unknown) & $64 / 283 / 76$ \\
\hline $\begin{array}{l}\text { Usage of other medication unrelated to breast } \\
\text { cancer (yes/no) }\end{array}$ & $126 / 297$ \\
\hline \multicolumn{2}{|l|}{ Clinical stage (UICC) } \\
\hline 0 & 54 \\
\hline I & 206 \\
\hline II $\mathrm{A}$ & 101 \\
\hline II $\mathrm{B}$ & 46 \\
\hline III A & 9 \\
\hline III B & 3 \\
\hline III C & 1 \\
\hline \multicolumn{2}{|l|}{ Histologic type } \\
\hline Noninvasive ductal carcinoma & 52 \\
\hline Invasive ductal carcinoma & 328 \\
\hline other & 43 \\
\hline \multicolumn{2}{|l|}{ Operation } \\
\hline Lumpectomy & 316 \\
\hline Quadrantectomy & 105 \\
\hline other & 2 \\
\hline Chemotherapy (yes/no) & $143 / 283$ \\
\hline pre-radiotherapy & 54 \\
\hline post-radiotherapy & 100 \\
\hline Endocrine therapy (yes/no ) & $313 / 98$ \\
\hline antiestrogen drug & 147 \\
\hline aromatase inhibitor & 164 \\
\hline Concurrent endocrine therapy (yes/no ) & $130 / 293$ \\
\hline antiestrogen drug & 47 \\
\hline aromatase inhibitor & 87 \\
\hline
\end{tabular}

Abbreviations : UICC $=$ International Union Against Cancer.

Table 2. Radiation therapy details ( $n=423$ breast)

\begin{tabular}{lr}
\hline Whole breast irradiation & \\
50 Gy/25 fraction & \\
Boost to tumor bed (yes/no) & $114 / 309$ \\
10 Gy/5 fraction & $405 / 18$ \\
Photon energy (4 MVX/6 MVX) & $422 / 1$ \\
Wedge filter (yes/no) & $0.8-3.2(1.8)$ \\
Central lung distance (cm) & $14.6-21.6(17.9)$ \\
Field length, long axis (cm) & $3.8-11.1(6.8)$ \\
Field length, short axis (cm) & 213 \\
Ipsilateral lung V20Gy (mean : $9.6 \%)$ & 210 \\
$1.8-9.6(\%)$ & 214 \\
$9.7-18.8(\%)$ & 209 \\
Ipsilateral lung V10Gy (mean : $12.2 \%)$ & 213 \\
$2.9-12.2(\%)$ & 210 \\
$12.3-22.3(\%)$ & 213 \\
Bilateral lung V20Gy (mean : $4.9 \%)$ & 210 \\
$0.8-4.9(\%)$ & \\
$4.9-14.2(\%)$ & \\
Bilateral V10Gy (mean : $6.2 \%)$ & \\
$1.3-6.2(\%)$ & \\
$6.3-17.5(\%)$ & \\
&
\end{tabular}


oral administration was started after radiotherapy in 183 cases. Anti-estrogen agents were administered in 147 cases and aromatase inhibitors (AIs) in 164 cases. In cases where medication was changed during the follow-up period, the medication that was administered initially was recorded. With regard to patient history, there were 6 cases of collagen disease, 30 cases of double cancer (18 cases of contralateral breast cancer), 3 cases of BCT experience for contralateral breast cancer and 73 cases of allergic disease. The use of treatment planning CT confirmed the absence of active pulmonary disease in any of these cases at the beginning of radiotherapy. All patients provided written informed consent before radiotherapy.

\section{Radiotherapy}

We used 4 or 6 million volt X-rays for the radiotherapy of remaining breasts, and conducted tangential irradiation of $50 \mathrm{~Gy} / 25$ fraction/5 weeks on all breasts in accordance with the Hinge method (using 4 or 6 million volt X-rays, wedge $15^{\circ}$ ). A $15^{\circ}$ wedge filter was used arbitrarily to equalize the dose distribution. The PRIMUS KD-2 (manufactured by Siemens/Toshiba) was used as a linear accelerator. With regard to 114 cases where the resection margins were positive, boost therapy of $10 \mathrm{~Gy} / 5$ fractions was added to the tumor beds using electron beams with appropriate energy.

\section{Follow-up}

We started the follow up examination with chest radiographs of every 3 months for 1 year after completion of radiotherapy. Based on previous study, radiation pneumonitis tends to occur shortly after the completion of radiotherapy (10). So, after the 1 year follow-up period using chest radiograph, patient's status were obtained by hospital records or interviews. When the chest radiographs showed an abnormal shadow, we performed the further examination using chest CT in order to evaluate the $\mathrm{RP}$ and confirm the grade of RP. The median period of the follow-up observation after completion of radiotherapy was 11.9 months (range : 2.9-41.6 months). The grade of RP was evaluated in accordance with version 3.0 of the NCI Common terminology Criteria for Adverse Events (CTC/AE ver. 3.0).

\section{Statistical analysis}

We analyzed risk factors for RP using univariate analysis (univariate Cox regression model) and multivariate analysis (Cox regression model). The variables analyzed were : patient age, history of allergic disease, the simultaneous use of systemic medicine (except medicine being used for the treatment of breast cancer), tumor location (every lesion located at the border between the outside and the inside of a breast was regarded as being inside), operative method, presence and timing of chemotherapy, whether the endocrine therapy was used during radiotherapy or in stages, whether the type of drug administered was an anti-estrogen agent or an AI, Xray energy, total irradiation dose, long and short axis length of the radiation field, central lung distance (CLD) measured from the axial image on the $\mathrm{XiO}$ and the volume receiving more than $20 \mathrm{~Gy}\left(\mathrm{~V}_{20 \mathrm{~Gy}}\right)$, as well as $\mathrm{V}_{10 \mathrm{~Gy}}$, of ipsilateral and bilateral lungs. Statistical significance was defined as $\mathrm{P}<0.05$ (Figs. $1,2)$.

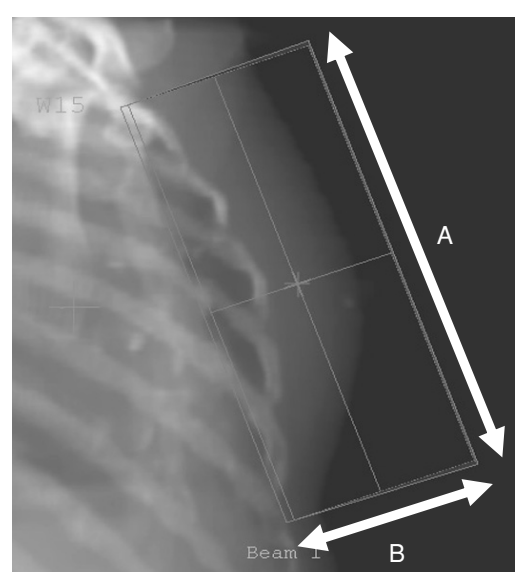

Fig. 1. Digitally reconstructed radiography image of the threedimensional treatment planning device in the radiation field A : long axis length of the radiation field $(\mathrm{cm}), \mathrm{B}$ : short axis length of the radiation field $(\mathrm{cm})$

At our hospital, the anterior border of the radiation field is set at a distance of $1.5 \mathrm{~cm}$ from the skin surface of the nipple base on the CT.

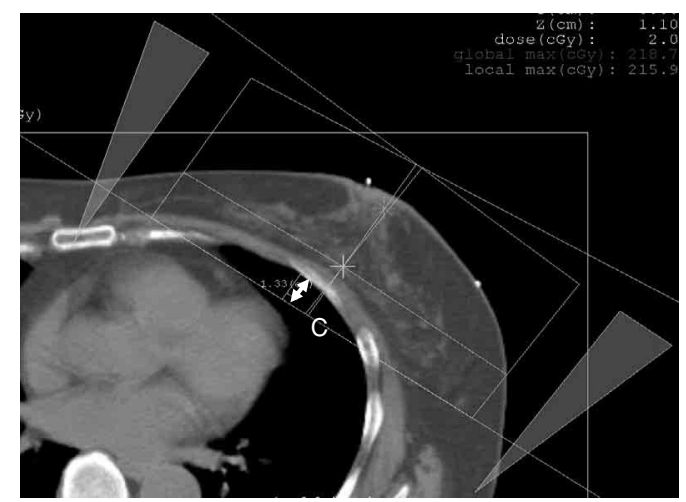

Fig. 2. The furthest distance between the posterior border of the radiation field and the chest wall was measured from the axial image of the treatment planning CT.

$\mathrm{C}$ : central lung distance (CLD) $(\mathrm{cm})$. 


\section{RESULTS}

\section{Cumulative incidence of $R P$}

Of the 423 breasts from 413 patients, 89 breasts from 84 cases (21\%) were diagnosed with RP, based on the chest radiograph. According to the evaluation of the grade of pneumonitis using CTC/AE ver. 3.0, there were 77 cases (18.2\%) of Grade $1 \mathrm{RP}, 10$ cases (2.3\%) of Grade 2 , and 2 cases $(0.5 \%)$ of Grade 3. The age of patients diagnosed with RP ranged from 25 to 80 years (median : 50). Thirty-six cases had $\mathrm{RP}$ in their left breasts and 53 in their right breasts. Fifteen cases (16.8\%) had a history of allergic disease and 17 (19.1\%) had a smoking history. In 32 cases $(35.9 \%)$, simultaneous medication was done, in addition to that for breast cancer treatment during radiotherapy. In 26 cases, lesions were found inside the breasts. In 60 cases, the T factor was T1 or less, while in 60 cases, the $\mathrm{N}$ factor was N0. According to the staging classification, 49 cases were diagnosed stage I or below. The operative method of 63 cases was lumpectomy. 72 cases were treated with endocrine therapy, of which 34 were treated during the irradiation period. With respect to factors regarding radiotherapy, 82 cases were treated with 4 MV X-rays, whereas 28 cases underwent boost irradiation. The long axis length of the radiation field was $14.6-21.6 \mathrm{~cm}$ (median : $17.9 \mathrm{~cm}$ ) and the short axis length was 3.8-11.1 cm (median : 6.8 $\mathrm{cm}$ ) ; the CLD was $0.8-3.2 \mathrm{~cm}$ (median : $1.8 \mathrm{~cm}$ ).

\section{Radiation-induced BOOP syndrome}

In the present analysis, radiation-induced BOOP syndrome was observed in $12 / 413$ cases (2.9\%). All cases of Grade $2 \mathrm{RP}$ or greater developed radiationinduced BOOP syndrome. The age of the patients ranged from 39 to 72 years (median : 50 years old). The clinical symptoms were fever (10 cases), coughing (12 cases), decrease in oxygen saturation (8 cases) and sense of dyspnea ( 8 cases). The timing of the pathogenesis was 2.5-23.1 months after the end of treatment (median : 3.6 months)., Abnormal shadows were observed on the chest radiographs taken 3 months after the completion of radiotherapy in all cases, except for one case, in which RP occurred after 23.1 months. Bronchoscopy was performed in 8 cases. The total cell number in bronchoalveolar lavage fluid was increased in $7 / 8$ cases. The transbronchial lung biopsy specimens in all 8 cases diagnosed pathologically as organized pneumonia. Of the 12 cases, steroid administration was implemented in 7 cases, resulting in rapid improvement of symptoms. However, recurrence of symptoms due to the decrease or suspension of the steroid administration was observed in 4 cases. The duration of the steroid administration was 5.8-41.4 months (median : 12.3 months) (Table 3).

A 39 year-old woman, in whom RP occurred after

Table 3. Clinical characteristics of 12 patients with radiation-induced BOOP syndrome

\begin{tabular}{|c|c|c|c|c|c|c|c|c|c|c|c|c|c|}
\hline Patient & $\begin{array}{l}\text { Age } \\
\text { (y) }\end{array}$ & side & $\begin{array}{l}\text { Endocrine } \\
\text { therapy }\end{array}$ & $\begin{array}{c}\text { Endocrine } \\
\text { therapy } \\
\text { (drug) }\end{array}$ & Chemotherapy & dose & $\begin{array}{l}\text { CLD } \\
(\mathrm{cm})\end{array}$ & $\begin{array}{l}\text { Short } \\
\text { axis } \\
(\mathrm{cm})\end{array}$ & $\begin{array}{c}\text { Ipsilateral } \\
\text { V20 Gy } \\
(\%)\end{array}$ & Steroid & $\begin{array}{c}\text { Steroid } \\
\text { (relapse) }\end{array}$ & $\begin{array}{l}\text { Onset, } \\
\text { After RT } \\
\text { (month) }\end{array}$ & $\begin{array}{l}\text { Duration } \\
\text { of steroid } \\
\text { (month) }\end{array}$ \\
\hline 1 & 40 & right & sequential & Tamoxifen & - & 60 & 2.4 & 8.2 & 12.05 & + & - & 3.2 & 5.8 \\
\hline 2 & 52 & left & sequential & Anastrozole & - & 50 & 2.0 & 6.6 & 13.93 & + & + & 4.4 & 12 \\
\hline 3 & 46 & right & none & - & - & 50 & 2.1 & 7.3 & 14.00 & - & - & 2.7 & - \\
\hline 4 & 53 & right & concurrent & Anastrozole & pre+post RT & 60 & 2.0 & 5.7 & 7.89 & - & - & 6.0 & - \\
\hline 5 & 48 & left & sequential & Tamoxifen & - & 50 & 1.7 & 6.7 & 8.88 & + & + & 2.5 & 41.1 \\
\hline 6 & 72 & right & sequential & Anastrozole & - & 60 & 2.0 & 6.4 & 8.35 & + & - & 6.9 & 11.1 \\
\hline 7 & 47 & right & sequential & Tamoxifen & - & 60 & 1.9 & 7.3 & 9.13 & - & - & 3.6 & - \\
\hline 8 & 59 & left & sequential & Anastrozole & - & 50 & 1.7 & 6.3 & 10.56 & + & + & 3.2 & 35.8 \\
\hline 9 & 69 & right & sequential & Anastrozole & - & 60 & 1.8 & 8.8 & 10.05 & + & + & 3.0 & 23.8 \\
\hline 10 & 59 & left & none & - & - & 50 & 2.3 & 6.7 & 12.14 & - & - & 3.7 & - \\
\hline 11 & 44 & right & concurrent & Tamoxifen & - & 60 & 2.1 & 6.6 & 8.74 & - & - & 3.7 & - \\
\hline 12 & 39 & left & concurrent & Tamoxifen & - & 50 & 1.7 & 5.7 & 9.05 & + & - & 23.1 & 12.2 \\
\hline
\end{tabular}

Abbreviations : $\mathrm{BOOP}=$ bronchiolitis obliterans organizing pneumonia ; $\mathrm{RT}=$ radiotherapy $\mathrm{CLD}=$ central lung distance 
23.1 months, had been taking immunosuppressive agents for a long period due to chronic rheumatoid arthritis. Moreover, radiation treatment planning CT did not show any lesions in her lung fields, and chest radiography taken 3 months after radiotherapy did not reveal any obvious abnormal shadows. Administration of the immunosuppressive agents was partially suspended 20 months after the completion of the radiotherapy. Moreover, an increased density area that was corresponding to the irradiation field, appeared in the lung field on the CT images at 22 months after radiotherapy. However, she complained of no symptoms at that time. Fever and coughing appeared 23 months after the completion of radiotherapy. Chest radiography revealed an infiltrative shadow expanding outside the radiation field. She was diagnosed with organized pneumonia using a bronchoscope, and was clinically diagnosed

A

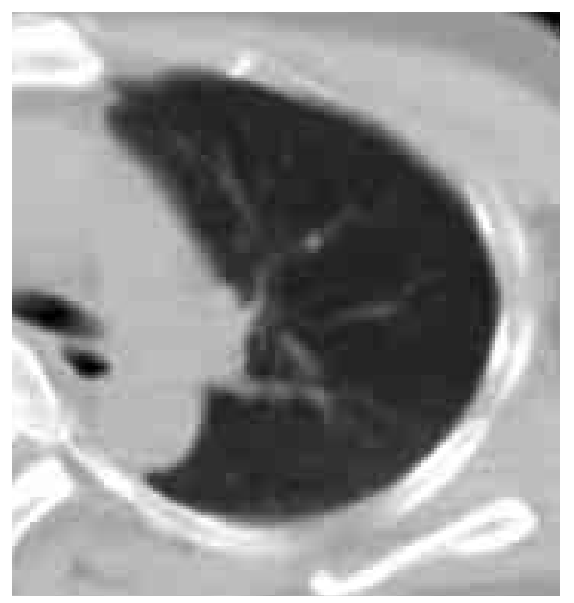

B

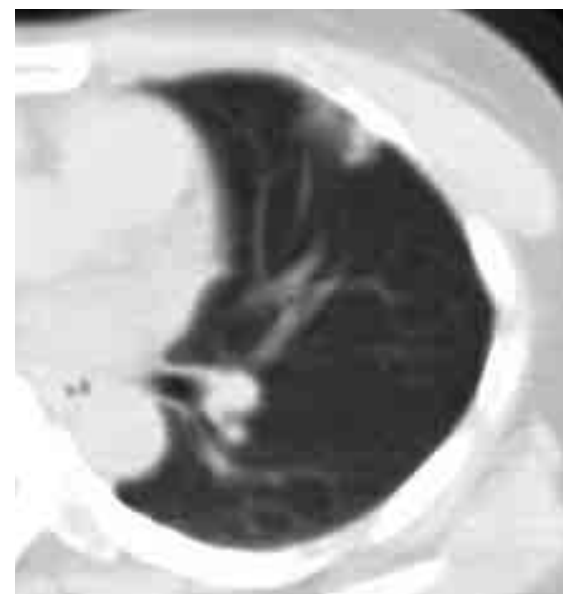

with radiation-induced BOOP syndrome. Therefore, we started steroid administration and her symptoms improved rapidly. Although this case did not match the criteria proposed by Crestani in terms of the timing of the pathogenesis(11), we extrapolated that RP can occur more than 1 year after irradiation provided that there are special conditions, such as the use of immunosuppressive agents, as in this case (Fig. 3).

\section{Risk factors for RP}

The univariate analysis detected significant differences in the following factors for incidence of RP of Grade 1 or greater : chemotherapy after radiotherapy $(p=0.005)$, chemotherapy in all the periods including before and after radiotherapy $(p=$ $0.001)$, short axis of the radiation field $(>6.8 \mathrm{~cm} ; p=$ 0.002), CLD ( $>1.8 \mathrm{~cm} ; p=0.002), \mathrm{N}$ factors $(\geq \mathrm{N} 1$;

$\mathrm{C}$

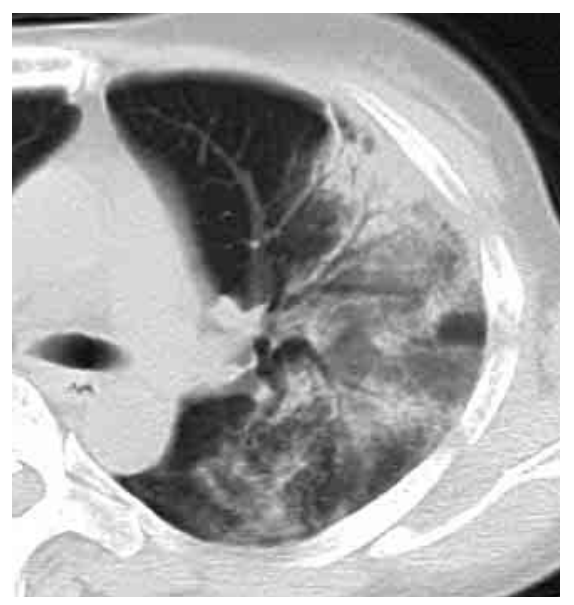

$\mathrm{D}$

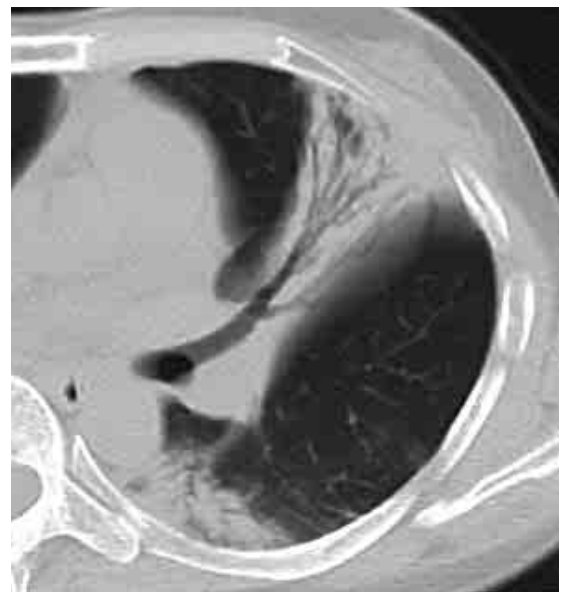

Fig. 3. A 39-year-old woman had been continuously taking immunosuppressive agents for the treatment of chronic rheumatoid arthritis. No abnormalities were recognized from the treatment planning CT before irradiation or from the chest radiograph taken 3 months after irradiation. Partial suspension of immunosuppressive agent administration 20 months after irradiation led to the detection of a localized concentration increasing region by chest CT conducted 22 months after irradiation. No symptoms were indicated at this point. Chest CT conducted 23 months after irradiation, however, revealed a clinical symptom consisting of infiltrative shadows and ground-glass opacity expanding outside the radiation field. They were diagnosed as organized pneumonia by bronchoscopy (A, B : CT performed 20 months after irradiation, C, D : CT performed 23 months after irradiation). 
$p=0.029)$, and use of AIs $(p=0.028)$ (Table 4, 5).

The cases of Grade 2 or greater were analyzed in the same manner. The univariate analysis detected significant differences only in CLD $(>1.8 \mathrm{~cm} ; p=$

Table 4. Univariate analysis of patient factors associated with Radiation pneumonitis

\begin{tabular}{|c|c|c|c|c|c|}
\hline Factor & & $\mathrm{RP}(\mathrm{n}=89)$ & $\mathrm{RR}$ & $p$-value & $95 \% \mathrm{CI}$ \\
\hline \multirow[t]{2}{*}{ Age } & $<50 \mathrm{y}$ & $33(191)$ & 0.76 & 0.20 & $0.49-1.2$ \\
\hline & $>51 \mathrm{y}$ & $56(232)$ & 1 & & \\
\hline \multirow[t]{2}{*}{ Side } & left & $36(201)$ & 0.73 & 0.15 & $0.48-1.1$ \\
\hline & right & $53(222)$ & 1 & & \\
\hline \multirow[t]{2}{*}{ Tumor factor } & T0,Tis,T1 & $60(301)$ & 0.84 & 0.43 & $0.54-1.3$ \\
\hline & $\mathrm{T} 2, \mathrm{~T} 3, \mathrm{~T} 4$ & $29(122)$ & 1 & & \\
\hline \multirow[t]{2}{*}{ Node factor } & N0 & $62(331)$ & 0.60 & 0.03 & $0.39-0.95$ \\
\hline & N1,N2,N3 & $27(92)$ & 1 & & \\
\hline \multirow[t]{2}{*}{ Clinical stage } & $<$ I stage & $49(263)$ & 0.73 & 0.14 & $0.48-1.1$ \\
\hline & $\geqq$ IIA stage & $40(160)$ & 1 & & \\
\hline \multirow[t]{2}{*}{ Location (region) } & inner side & $26(161)$ & 0.65 & 0.06 & $0.41-1.0$ \\
\hline & $\begin{array}{l}\text { outer side } \\
\text { unknown }\end{array}$ & $\begin{array}{c}63(259) \\
0(3)\end{array}$ & 1 & & \\
\hline \multirow{2}{*}{ Operation } & lumpectomy & $63(316)$ & 0.76 & 0.24 & $0.48-1.2$ \\
\hline & quadrantectomy & $26(105)$ & 1 & & \\
\hline \multirow[t]{2}{*}{ Allergic disease } & $(+)$ & $15(73)$ & 0.96 & 0.89 & $0.55-1.7$ \\
\hline & $(-)$ & $74(350)$ & 1 & & \\
\hline \multirow[t]{2}{*}{ Usage of other medication unrelated to breast cancer } & $(+)$ & $32(126)$ & 1.23 & 0.34 & $0.80-1.9$ \\
\hline & $(-)$ & $57(297)$ & 1 & & \\
\hline \multirow[t]{3}{*}{ Smoking status } & $(+)$ & $17(64)$ & 1.14 & 0.70 & $0.65-1.9$ \\
\hline & $(-)$ & $66(283)$ & 1 & & \\
\hline & unknown & $0(76)$ & & & \\
\hline \multirow[t]{2}{*}{ Chemotherapy } & $(+)$ & $44(143)$ & 1.98 & $<0.01$ & $1.3-3.0$ \\
\hline & $(-)$ & $45(280)$ & 1 & & \\
\hline \multirow[t]{2}{*}{ chemotherapy (pre-RT) } & $(+)$ & $14(54)$ & 1.27 & 0.41 & $0.72-2.3$ \\
\hline & $(-)$ & $75(369)$ & 1 & & \\
\hline \multirow[t]{2}{*}{ chemotherapy (post-RT) } & $(+)$ & $32(100)$ & 1.87 & $<0.01$ & $1.2-2.9$ \\
\hline & $(-)$ & $57(323)$ & 1 & & \\
\hline \multirow[t]{3}{*}{ Endocrine therapy } & $(+)$ & $72(313)$ & 1.39 & 0.23 & $0.82-2.4$ \\
\hline & $(-)$ & $17(98)$ & 1 & & \\
\hline & unknown & $0(12)$ & & & \\
\hline \multirow[t]{2}{*}{ antiestrogen drug } & $(+)$ & $26(148)$ & 0.79 & 0.32 & $0.50-1.3$ \\
\hline & $(-)$ & $63(275)$ & 1 & & \\
\hline \multirow[t]{2}{*}{ aromatase inhibitor } & $(+)$ & $45(164)$ & 1.59 & 0.03 & $1.1-2.4$ \\
\hline & $(-)$ & $44(259)$ & 1 & & \\
\hline \multirow[t]{2}{*}{ (Concurrent endocrine therapy) } & concurrent & $34(130)$ & 1.34 & 0.20 & $0.85-2.1$ \\
\hline & sequential & $38(183)$ & 1 & & \\
\hline \multirow[t]{2}{*}{ antiestrogen drug } & concurrent & $10(47)$ & 1.08 & 0.81 & $0.56-2.1$ \\
\hline & other & $79(376)$ & 1 & & \\
\hline \multirow[t]{2}{*}{ aromatase inhibitor } & concurrent & $24(83)$ & 1.59 & 0.05 & $0.99-2.5$ \\
\hline & other & $65(340)$ & 1 & & \\
\hline
\end{tabular}

Abbreviations : $\mathrm{RP}=$ radiation pneumonitis ; $\mathrm{RT}=$ radiotherapy ; $\mathrm{CLD}=$ central lung distance $; \mathrm{RR}=$ relative risk ; $\mathrm{CI}=$ confidence interval.

Table 5. Univariate analysis of radiotherapy factors associated with Radiation pneumonitis

\begin{tabular}{|c|c|c|c|c|c|}
\hline Factor & & $\mathrm{RP}(\mathrm{n}=89)$ & $\mathrm{RR}$ & $p$-value & $95 \% \mathrm{CI}$ \\
\hline \multirow[t]{2}{*}{ Energy } & 4 MVX & $82(405)$ & 0.58 & 0.17 & $0.27-1.3$ \\
\hline & 6 MVX & $7(18)$ & 1 & & \\
\hline \multirow[t]{2}{*}{ Radiation dose } & $50 \mathrm{~Gy}$ & $61(309)$ & 0.76 & 0.23 & $0.49-1.2$ \\
\hline & $60 \mathrm{~Gy}$ & $25(114)$ & 1 & & \\
\hline \multirow[t]{2}{*}{ Field length (long axis) (mean : $17.9 \mathrm{~cm}$ ) } & $14.6-17.9$ & $45(212)$ & 1.01 & 0.97 & $0.67-1.5$ \\
\hline & $18.0-21.6$ & $44(211)$ & 1 & & \\
\hline \multirow[t]{2}{*}{ Field length (short axis) (mean : $6.8 \mathrm{~cm}$ ) } & $3.8-6.8$ & $33(217)$ & 0.51 & $<0.01$ & $0.33-0.79$ \\
\hline & $6.8-11.1$ & $56(206)$ & 1 & & \\
\hline \multirow[t]{2}{*}{ CLD (mean : $1.8 \mathrm{~cm}$ ) } & $0.8-1.8$ & $42(222)$ & 0.51 & $<0.01$ & $0.35-0.80$ \\
\hline & 1.9-3.1 & $47(112)$ & 1 & & \\
\hline \multirow{2}{*}{ Ipsilateral lung V20Gy (mean : 9.6\%) } & $1.8-9.6(\%)$ & $36(213)$ & 0.67 & 0.13 & $0.48-1.1$ \\
\hline & $9.7-18.8(\%)$ & $53(210)$ & 1 & & \\
\hline \multirow[t]{2}{*}{ Ipisilateral lung $\mathrm{V}_{10 \mathrm{~Gy}}$ (mean : 12.2\%) } & 2.9-12.2 (\%) & $37(214)$ & 0.69 & 0.09 & $0.45-1.1$ \\
\hline & $12.3-22.3(\%)$ & $52(209)$ & 1 & & \\
\hline \multirow[t]{2}{*}{ Bilateral lung $\mathrm{V}_{20 \mathrm{~Gy}}$ (mean : 4.9\%) } & 0.8-4.9 (\%) & $38(213)$ & 0.75 & 0.17 & $0.49-1.1$ \\
\hline & $4.9-14.2(\%)$ & $51(210)$ & 1 & & \\
\hline \multirow[t]{2}{*}{ Bilateral ling $\mathrm{V}_{10 \mathrm{~Gy}}$ (mean : .2\%) } & 1.3-6.2 (\%) & $39(213)$ & 0.77 & 0.23 & $0.51-1.2$ \\
\hline & $6.3-17.5(\%)$ & $50(210)$ & 1 & & \\
\hline
\end{tabular}

Abbreviations : $\mathrm{RP}=$ radiation pneumonitis ; $\mathrm{RT}=$ radiotherapy ; $\mathrm{CLD}=$ central lung distance $; \mathrm{RR}=$ relative risk ; $\mathrm{CI}=$ confidence interval. 
0.043) and in the operative method (lumpectomy ; $p=0.007)$. Other than these factors, the patients who underwent chemotherapy $(p=0.090)$ and the patients who received radiation dose of $60 \mathrm{~Gy}(p=0.080)$ tended to appear RP, but it was not statistically significant (Table 6,7).

Table 6. Univariate analysis of patient factors associated with RP of Grade 2 or greater, diagnosed as radiation-induced BOOP syndrome

\begin{tabular}{|c|c|c|c|c|c|}
\hline Factor & & BOOP $(n=12)$ & $\mathrm{RR}$ & $p$-value & $95 \% \mathrm{CI}$ \\
\hline \multirow[t]{2}{*}{ Age } & $<50 \mathrm{y}$ & $6(191)$ & 1.30 & 0.65 & $0.42-4.0$ \\
\hline & $>51 \mathrm{y}$ & $6(232)$ & 1 & & \\
\hline \multirow[t]{2}{*}{ Side } & left & $5(201)$ & 0.77 & 0.66 & $0.24-2.4$ \\
\hline & right & $7(222)$ & 1 & & \\
\hline \multirow[t]{2}{*}{ Tumor factor } & T0,Tis,T1 & $9(301)$ & 1.25 & 0.74 & $0.34-4.6$ \\
\hline & $\mathrm{T} 2, \mathrm{~T} 3, \mathrm{~T} 4$ & $3(122)$ & 1 & & \\
\hline \multirow[t]{2}{*}{ Node factor } & N0 & 11(331) & 3.20 & 0.27 & $0.41-24.8$ \\
\hline & $\mathrm{N} 1, \mathrm{~N} 2, \mathrm{~N} 3$ & $1(92)$ & 1 & & \\
\hline \multirow[t]{2}{*}{ Clinical stage } & $<$ I stage & $9(263)$ & 1.87 & 0.35 & $0.51-6.9$ \\
\hline & $\geqq$ IIA stage & $3(160)$ & 1 & & \\
\hline \multirow[t]{3}{*}{ Location (region) } & inner side & $3(161)$ & 0.54 & 0.35 & $0.15-2.0$ \\
\hline & outer side & $9(259)$ & 1 & & \\
\hline & unknown & $0(3)$ & & & \\
\hline \multirow[t]{3}{*}{ Operation } & lumpectomy & $5(316)$ & 0.21 & $<0.01$ & $0.07-0.65$ \\
\hline & quadrantectomy & $7(105)$ & 1 & & \\
\hline & other & $0(2)$ & & & \\
\hline \multirow[t]{2}{*}{ Allergic disease } & $(+)$ & $4(73)$ & 2.35 & 0.16 & $0.71-7.8$ \\
\hline & $(-)$ & $8(350)$ & 1 & & \\
\hline \multirow[t]{2}{*}{ Usage of other medication unrelated to breast cancer } & $(+)$ & $3(126)$ & 0.73 & 0.63 & $0.20-2.7$ \\
\hline & $(-)$ & $9(297)$ & 1 & & \\
\hline \multirow[t]{3}{*}{ Smoking status } & $(+)$ & $1(64)$ & 0.38 & 0.36 & $0.05-3.0$ \\
\hline & $(-)$ & $11(283)$ & 1 & & \\
\hline & unknown & $0(76)$ & & & \\
\hline \multirow[t]{2}{*}{ Chemotherapy } & $(+)$ & $1(143)$ & 0.17 & 0.09 & $0.02-1.3$ \\
\hline & $(-)$ & $11(280)$ & 1 & & \\
\hline \multirow[t]{2}{*}{ chemotherapy (pre-RT) } & $(+)$ & $1(54)$ & 0.63 & 0.66 & $0.08-4.9$ \\
\hline & $(-)$ & 11(369) & 1 & & \\
\hline \multirow[t]{2}{*}{ chemotherapy (post-RT) } & $(+)$ & $1(100)$ & 0.27 & 0.21 & $0.04-2.1$ \\
\hline & $(-)$ & 11(323) & 1 & & \\
\hline \multirow[t]{3}{*}{ Endocrine therapy } & $(+)$ & $10(313)$ & 1.55 & 0.54 & $0.34-7.1$ \\
\hline & $(-)$ & $2(98)$ & 1 & & \\
\hline & unknown & $0(12)$ & & & \\
\hline \multirow[t]{2}{*}{ antiestrogen drug } & $(+)$ & $5(148)$ & 1.32 & 0.63 & $0.42-4.2$ \\
\hline & $(-)$ & $7(275)$ & 1 & & \\
\hline \multirow[t]{2}{*}{ aromatase inhibitor } & $(+)$ & $5(164)$ & 1.06 & 0.92 & $0.34-3.3$ \\
\hline & $(-)$ & $7(259)$ & 1 & & \\
\hline \multirow[t]{2}{*}{ (Concurrent endocrine therapy) } & concurrent & $4(130)$ & 1.02 & 0.98 & $0.29-2.6$ \\
\hline & sequential & $6(183)$ & 1 & & \\
\hline \multirow[t]{2}{*}{ antiestrogen drug } & concurrent & $2(47)$ & 1.70 & 0.50 & $0.37-7.7$ \\
\hline & other & $10(376)$ & 1 & & \\
\hline \multirow[t]{2}{*}{ aromatase inhibitor } & concurrent & $2(83)$ & 0.82 & 0.80 & $0.18-3.7$ \\
\hline & other & $10(340)$ & 1 & & \\
\hline
\end{tabular}


Table 7. Univariate analysis of radiotherapy factors associated with RP of Grade 2 or greater, diagnosed as radiation-induced BOOP syndrome

\begin{tabular}{|c|c|c|c|c|c|}
\hline Factor & & BOOP $(n=12)$ & $\mathrm{RR}$ & $p$-value & $95 \% \mathrm{CI}$ \\
\hline \multirow[t]{2}{*}{ Energy } & $4 \mathrm{MVX}$ & $12(405)$ & & & \\
\hline & $6 \mathrm{MVX}$ & $0(18)$ & & & \\
\hline \multirow[t]{2}{*}{ Radiation dose } & $50 \mathrm{~Gy}$ & $6(309)$ & 0.36 & 0.08 & $0.12-1.1$ \\
\hline & $60 \mathrm{~Gy}$ & $6(114)$ & 1 & & \\
\hline \multirow[t]{2}{*}{ Field length (long axis) (mean : $17.9 \mathrm{~cm}$ ) } & $14.6-17.9$ & $6(212)$ & 1.00 & 0.99 & $0.32-3.1$ \\
\hline & $18.0-21.6$ & $6(211)$ & 1 & & \\
\hline \multirow[t]{2}{*}{ Field length (short axis) (mean : $6.8 \mathrm{~cm}$ ) } & $3.8-6.8$ & $8(217)$ & 1.76 & 0.36 & $0.53-5.9$ \\
\hline & $6.8-11.1$ & $4(206)$ & 1 & & \\
\hline \multirow[t]{2}{*}{ CLD (mean : $1.8 \mathrm{~cm}$ ) } & $0.8-1.8$ & $4(222)$ & 0.29 & 0.04 & $0.09-0.96$ \\
\hline & $1.9-3.1$ & $8(112)$ & 1 & & \\
\hline \multirow[t]{2}{*}{ Ipsilateral lung V20Gy (mean : 9.6\%) } & $1.8-9.6(\%)$ & $7(213)$ & 1.02 & 0.97 & $0.32-3.2$ \\
\hline & $9.7-18.8(\%)$ & $5(210)$ & 1 & & \\
\hline \multirow[t]{2}{*}{ Ipisilateral lung $\mathrm{V}_{10 \mathrm{~Gy}}($ mean : $12.2 \%)$} & $2.9-12.2(\%)$ & $6(214)$ & 1.01 & 0.99 & $0.33-3.1$ \\
\hline & $12.3-22.3(\%)$ & $6(209)$ & 1 & & \\
\hline \multirow[t]{2}{*}{ Bilateral lung $V_{20 G y}($ mean : 4.9\%) } & $0.8-4.9(\%)$ & $6(213)$ & 1.04 & 0.95 & $0.33-3.2$ \\
\hline & $4.9-14.2(\%)$ & $6(210)$ & 1 & & \\
\hline \multirow[t]{2}{*}{ Bilateral ling $\mathrm{V}_{10 \mathrm{~Gy}}$ (mean : 6.2\%) } & $1.3-6.2(\%)$ & $6(213)$ & 1.04 & 0.95 & $0.33-3.2$ \\
\hline & $6.3-17.5(\%)$ & $6(210)$ & 1 & & \\
\hline
\end{tabular}

Abbreviations : $\mathrm{BOOP}=$ bronchiolitis obliterans organizing pneumonia $; \mathrm{RT}=$ radiotherapy $; \mathrm{CLD}=$ central lung distance ; $\mathrm{RR}=$ relative risk ; $\mathrm{CI}=$ confidence interval.

The multivariate analysis detected significant differences in the following factors for incidence of Grade 1 or greater RP : short axis length $(>6.8 \mathrm{~cm}$; $p=0.024)$ and CLD (>1.8 cm; $p=0.021)$ (Table 8).
The multivariate analysis of the RP cases of Grade 2 or greater detected a significant difference only in CLD (> $1.8 \mathrm{~cm} ; p=0.027$ ) (Table 9).

Table 8. Multivariate analysis of factors associated with RP

\begin{tabular}{|c|c|c|c|c|c|}
\hline Factor & & $\mathrm{RP}(\mathrm{n}=89)$ & $\mathrm{RR}$ & $p$-value & $95 \% \mathrm{CI}$ \\
\hline \multirow[t]{2}{*}{ Node factor } & No & $62(331)$ & 1.08 & 0.78 & $0.61-1.9$ \\
\hline & N1,N2,N3 & $27(92)$ & 1 & & \\
\hline \multirow[t]{3}{*}{ Location (region) } & inner side & $26(161)$ & 0.76 & 0.25 & $0.48-1.2$ \\
\hline & outer side & $63(259)$ & 1 & & \\
\hline & unknown & $0(3)$ & & & \\
\hline \multirow[t]{2}{*}{ Chemotherapy } & $(+)$ & $44(143)$ & 1.70 & 0.16 & $0.81-3.6$ \\
\hline & $(-)$ & $45(280)$ & 1 & & \\
\hline \multirow[t]{2}{*}{ chemotherapy (post-RT) } & $(+)$ & $32(100)$ & 1.32 & 0.45 & $0.65-2.7$ \\
\hline & $(-)$ & $57(323)$ & 1 & & \\
\hline \multirow[t]{2}{*}{ aromatase inhibitor } & $(+)$ & $45(164)$ & 1.39 & 0.24 & $0.80-2.4$ \\
\hline & $(-)$ & $44(259)$ & 1 & & \\
\hline \multirow[t]{2}{*}{ aromatase inhibitor } & concurrent & $24(83)$ & 1.31 & 0.41 & $0.69-2.5$ \\
\hline & other & $65(340)$ & 1 & & \\
\hline \multirow[t]{2}{*}{ Field length (short axis) (mean : $6.8 \mathrm{~cm}$ ) } & $3.8-6.8$ & $33(217)$ & 0.60 & 0.02 & $0.39-0.94$ \\
\hline & $6.8-11.1$ & $56(206)$ & 1 & & \\
\hline \multirow[t]{2}{*}{ CLD (mean : $1.8 \mathrm{~cm})$} & $0.8-1.8$ & $42(222)$ & 0.58 & 0.02 & $0.36-0.92$ \\
\hline & $1.9-3.1$ & $47(112)$ & 1 & & \\
\hline \multirow[t]{2}{*}{ Ipsilateral lung V20Gy (mean : 9.6\%) } & $1.8-9.6(\%)$ & $36(213)$ & 0.89 & 0.63 & $0.56-1.4$ \\
\hline & $9.7-18.8(\%)$ & $53(210)$ & 1 & & \\
\hline
\end{tabular}

Abbreviations : $\mathrm{RP}=$ radiation pneumonitis $; \mathrm{RT}=$ radiotherapy $; \mathrm{CLD}=$ central lung distance $; \mathrm{RR}=$ relative risk ; $\mathrm{CI}=$ confidence interval. 
Table 9. Multivariate analysis of factors associated with Grade2 or greater pneumonitis, diagnosed as radiation-induced BOOP syndrome

\begin{tabular}{|c|c|c|c|c|c|}
\hline Factor & & BOOP $(\mathrm{n}=12)$ & $\mathrm{RR}$ & $p$-value & $95 \% \mathrm{CI}$ \\
\hline \multirow[t]{2}{*}{ Chemotherapy } & $(+)$ & $1(143)$ & 0.17 & 0.09 & $0.02-1.3$ \\
\hline & $(-)$ & $11(280)$ & 1 & & \\
\hline \multirow[t]{2}{*}{ Radiation dose } & 50 Gy & $6(309)$ & 0.44 & 0.17 & $0.14-1.4$ \\
\hline & $60 \mathrm{~Gy}$ & $6(114)$ & 1 & & \\
\hline \multirow[t]{2}{*}{ Field length (short axis) (mean : $6.8 \mathrm{~cm}$ ) } & $3.8-6.8$ & $8(217)$ & 1.55 & 0.49 & $0.45-5.3$ \\
\hline & $6.8-11.1$ & $4(206)$ & 1 & & \\
\hline \multirow[t]{2}{*}{ CLD (mean : $1.8 \mathrm{~cm}$ ) } & $0.8-1.8$ & $4(222)$ & 0.24 & 0.03 & $0.07-0.85$ \\
\hline & $1.9-3.1$ & $8(112)$ & 1 & & \\
\hline \multirow[t]{2}{*}{ Ipsilateral lung $\mathrm{V}_{20 \mathrm{~Gy}}$ (mean : 9.6\%) } & $1.8-9.6(\%)$ & $7(213)$ & 1.64 & 0.43 & $0.48-5.6$ \\
\hline & $9.7-18.8(\%)$ & $5(210)$ & 1 & & \\
\hline
\end{tabular}

Abbreviations : $\mathrm{BOOP}=$ bronchiolitis obliterans organizing pneumonia $; \mathrm{RT}=$ radiotherapy $; \mathrm{CLD}=$ central lung distance ; $\mathrm{RR}=$ relative risk ; $\mathrm{CI}=$ confidence interval.

\section{DISCUSSION}

The frequency of pulmonary damage caused by radiotherapy following breast cancer surgery, including RP occurring at an early time after irradiation and fibrosis occurring at a late time, varies greatly from $0.9 \%$ to $85 \%$, depending on reports. Naturally, the detection rates differ between chest radiography and chest CT. According to a previous report, CT evaluation 3 months after radiotherapy showed a frequency of Grade 1 or greater RP of 37\% (14). HRCT evaluation, 4 months after radiotherapy, also detected changes in pulmonary parenchyma within radiation fields in $85 \%$ of cases(7). In general, the frequency of RP evaluated by chest radiograph is 0.9 $47 \%$. In the present study, $21 \%$ of patients showed RP on the chest radiograph at 3 months after completion of radiotherapy. The previous reports, however, also involve local-regional radiotherapy on supraclavicular and axilla fields, as well as chest-wall irradiation after mastectomy and differ slightly from our study with respect to the subjects $(2,6,14,16,17)$. BOOP was reported in 1985 by Epler, et al. It presents symptoms such as coughing, sense of dyspnea, fever and chest pain. On images, it shows patchy consolidation accompanied by ground-glass opacities. Histological characteristics include polypoid masses of granulation tissue in the lumens of small airways, alveolar ducts and some alveoli. Although it can be classified into idiopathic and secondary, the majority of cases are usually idiopathic. The frequency of radiation-induced BOOP syndrome following breast-conserving surgery of breast cancer has been reported as 2.3\% (15), 2.4\% (13) and 2.5\% (12). In the present study, a similar incidence of
$2.9 \%$ was obtained as a result. It included one atypical case among the 12 cases in which clinically problematic RP of Grade 2 or greater presented. Apart from this case, however, clinical symptoms requiring treatment did not appear before the period of 2.5-6.9 months after radiotherapy. In all cases, slight abnormal shadows can be detected on the chest radiographs at the 3 -month after radiotherapy. It is considered that $\mathrm{RP}$ and radiation-induced BOOP syndrome are two different clinical conditions. RP results from the direct influence of irradiation, whereas radiation-induced BOOP syndrome is believed to be caused by autoimmunization induced indirectly by irradiation $(10-13,18,19)$. Nevertheless, at the onset of radiation-induced BOOP syndrome, infiltrative shadows resembling ground-glass opacity, in areas corresponding to radiation fields, begin to appear. They then develop into infiltrative shadows expanding outside the radiation field areas. In the present study, there were hardly any clinical symptoms 3 months after radiotherapy. However, the next follow-up observations indicated 7 cases of exacerbated symptoms of Grade 2 or greater, despite the fact that the CT taken at 3 months after radiotherapy was only able to detect localized shadows within the radiation field. This led us to believe that conducting an evaluation 3 months after radiotherapy was crucial.

There have been several reports analyzing the factors concerning pathogenesis of RP and radiation-induced BOOP syndrome caused by irradiation following breast cancer surgery. There have been 4 reports investigating radiation-induced BOOP syndrome caused by irradiation after conserving surgery $(12,13,15,20)$. However, no reports have 
been published investigating the effects of patient background and treatment regimens on the pathogenesis of Grade $1 \mathrm{RP}$. In the present study, we only analyzed cases in which whole breast irradiation had been performed and in which all radiotherapy technical factors had been evaluated. As for the relationship between patient characteristics and RP, although patient age has often been cited as a risk factor $(2,14,20-24)$, no such relationship was established in the present study. In agreement with previous reports, smoking history and the simultaneous use of other medication were not identified as factors influencing risk of $\mathrm{RP}(3,20,25)$. The use of radiotherapy and chemotherapy simultaneously increases the risk of $\mathrm{RP}(9,17)$, however, the risk induced by the use of chemotherapy in stages varies greatly among reports $(15,20,26,27)$. Our univariate analysis also suggested that adjuvant chemotherapy increases the risk of RP.

There are numerous reports indicating the involvement of anti-estrogen agents in the pathogenesis of RP, especially tamoxifen $(15,21,22,28,29)$. In the present study, however, anti-estrogen agents were not a significant variable for $\mathrm{RP}$, whereas a correlation was found with the use of AIs. Increasing of RP patients who have used AIs might be related to age because AIs are generally used in postmenopausal women. With regard to radiotherapy factors, breast and regional irradiation including supraclavicular and axilla (local-regional radiotherapy) is considered to result in a higher frequency of RP compared with whole breast irradiation (local radiotherapy) $(2,6,14,16,17)$. Lind, et al. have reported that the risk of RP was significantly higher in localregional radiotherapy (4.1\%) compared with local radiotherapy (0.9\%) (16). Kahan (14), likewise, has reported that the incidence of $\mathrm{RP}$ and fibrosis were 2.5 times higher in regional irradiation. $\mathrm{V}_{20 \mathrm{~Gy}}$ was reported to correlate with lung volume; the more $\mathrm{V}_{\text {20Gy }}$ increased, the higher the incidence of RP (2, $14,23)$. So we investigated only cases of breast irradiation, the frequency of $\mathrm{RP}$ had no significant statistically correlation with $\mathrm{V}_{20 \mathrm{~Gy}}$ as well as $\mathrm{V}_{10 \mathrm{~Gy}}$ in the present study. Previous reports, on the other hand, took into consideration the regional irradiation, leading to the speculation that significant differences were obtained due to the high influence of the regional irradiation. There have also been several reports indicating the involvement of CLD with RP (8, 9). Fernando, et al. (9) have reported the frequency of RP as $4.6 \%$ in cases where CLD was $3 \mathrm{~cm}$ or more. The present study also showed that CLD of
$1.9 \mathrm{~cm}$ or more increased the risk of $\mathrm{RP}$ and radiation-induced BOOP syndrome. Another result of the present study was that the short axis length of the radiation field correlated with RP in the univariate and multivariate analyses. Although there have been reports examining the long axis length of radiation fields (15), it is difficult to compare the short axis with other analyses owing to different operational criteria among institutions. Nevertheless, RP is considerably affected by the lung volume to be irradiated, in the same way as CLD. The radiation dose exhibited no significant difference, albeit a tendency to correlate with the radiation-induced BOOP syndrome was observed. There are numerous reports investigating risk factors for the $\mathrm{RP}$ and radiationinduced BOOP syndrome. Though, in our study, there are no significant differences with previously suggested factors, such as age and the simultaneous use of hormonal treatment. In multivariate analysis, setting of the radiation field, such as CLD and short axis length of the radiation field, was an important factor. As for the timing of the RP, RP and radiationinduced BOOP syndrome that required treatment were detected on the chest radiography 3 months after the treatment, suggesting that evaluation at this stage is crucial. These results are suggesting that we need to give careful attention to occurrence of $\mathrm{RP}$ in around 3 months after completion of RT.

\section{CONCLUSIONS}

In the tangential irradiation of whole breast after breast conserving surgery, the significant risk factors of RP are CLD $(>1.8 \mathrm{~cm})$ and the short axis length of the radiation field, as determined by multivariate analysis. The pathogenesis of radiation-induced BOOP syndrome significantly correlated with CLD. Therefore, the lung volume within the radiation field proved to be a significant risk factor for $\mathrm{RP}$ and radiation-induced BOOP syndrome. Regarding follow-up after irradiation, the evaluation of chest radiography 3 months after the end of irradiation is postulated to be crucial.

\section{REFERENCES}

1. Clarke M, Collins R, Darby S, Davies C, Elphinstone P, Evans E, Godwin J, Gray R, Hicks C, James S, MacKinnon E, McGale P, McHugh T, Peto R, Taylor C, Wang Y : Effects 
of radiotherapy and of differences in the extent of surgery for early breast cancer on local recurrence and 15-year survival : an overview of the randomised trials. Lancet $366: 2087-2106$, 2005

2. Lind PA, Wennberg B, Gagliardi G, Fornander $\mathrm{T}$ : Pulmonary complications following different radiotherapy techniques for breast cancer, and the association to irradiated lung volume and dose. Breast Cancer Res Treat $68: 199-210$, 2001

3. Holli K, Pitkanen M, Jarvenpaa R, Rajala J, Lahtela S, Hyodynmaa S, Ojala A : Early skin and lung reactions in breast cancer patients after radiotherapy : prospective study. Radiother Oncol 64 : 163-169, 2002

4. Zissiadis Y, Langlands AO, Barraclough B, Boyages J : Breast conservation : long-term results from Westmead Hospital. Aust N Z J Surg $67: 313-319,1997$

5. Fung MC, Schultz DJ, Solin LJ : Early-stage bilateral breast cancer treated with breast-conserving surgery and definitive irradiation : the University of Pennsylvania experience. Int J Radiat Oncol Biol Phys 38 : 959-967, 1997

6. Wennberg B, Gagliardi G, Sundbom L, Svane G, Lind P : Early response of lung in breast cancer irradiation : radiologic density changes measured by $\mathrm{CT}$ and symptomatic radiation pneumonitis. Int J Radiat Oncol Biol Phys 52 : 1196-1206, 2002

7. Ogoh E, Fujimoto K, Meno S, Uchida M, Joh S, Tabuchi E, Toda Y, Onizuka Y, Nishimura $\mathrm{H}$, Hayabuchi N : [HR-CT evaluation of lung parenchymal alterations in patients following breast conservation therapy]. Nippon Igaku Hoshasen Gakkai Zasshi 58 : 331-337, 1998 (in Japanese)

8. Lind PA, Gagliardi G, Wennberg B, Fornander $\mathrm{T}$ : A descriptive study of pulmonary complications after postoperative radiation therapy in node-positive stage II breast cancer. Acta Oncol $36: 509-515,1997$

9. Fernando IN, Powles TJ, Ashley S, Grafton D, Harmer CL, Ford HT : An acute toxicity study on the effects of synchronous chemotherapy and radiotherapy in early stage breast cancer after conservative surgery. Clin Oncol (R Coll Radiol) $8: 234-238,1996$

10. Abratt RP, Morgan GW : Lung toxicity following chest irradiation in patients with lung cancer. Lung Cancer 35 : 103-109, 2002
11. Crestani B, Valeyre D, Roden S, Wallaert B, Dalphin JC, Cordier JF : Bronchiolitis obliterans organizing pneumonia syndrome primed by radiation therapy to the breast. The Groupe d'Etudes et de Recherche sur les Maladies Orphelines Pulmonaires (GERM“O”P). Am J Respir Crit Care Med 158 : 1929-1935, 1998

12. Takigawa N, Segawa Y, Saeki T, Kataoka M, Ida M, Kishino D, Fujiwara K, Ohsumi S, Eguchi K, Takashima S : Bronchiolitis obliterans organizing pneumonia syndrome in breastconserving therapy for early breast cancer : radiation-induced lung toxicity. Int J Radiat Oncol Biol Phys 48 : 751-755, 2000

13. Miwa S, Morita S, Suda T, Suzuki K, Hayakawa $\mathrm{H}$, Chida K, Nakamura $\mathrm{H}$ : The incidence and clinical characteristics of bronchiolitis obliterans organizing pneumonia syndrome after radiation therapy for breast cancer. Sarcoidosis Vasc Diffuse Lung Dis 21 : 212-218, 2004

14. Kahan Z, Csenki M, Varga Z, Szil E, Cserhati A, Balogh A, Gyulai Z, Mandi Y, Boda K, Thurzo L: The risk of early and late lung sequelae after conformal radiotherapy in breast cancer patients. Int J Radiat Oncol Biol Phys $68: 673-681,2007$

15. Katayama N, Sato S, Katsui K, Takemoto M, Tsuda T, Yoshida A, Morito T, Nakagawa T, Mizuta A, Waki T, Niiya H, Kanazawa S : Analysis of Factors Associated with Radiation-Induced Bronchiolitis Obliterans Organizing Pneumonia Syndrome After Breast-Conserving Therapy. Int J Radiat Oncol Biol Phys : 2008

16. Lind PA, Marks LB, Hardenbergh PH, Clough R, Fan M, Hollis D, Hernando ML, Lucas D, Piepgrass A, Prosnitz LR : Technical factors associated with radiation pneumonitis after local +/- regional radiation therapy for breast cancer. Int J Radiat Oncol Biol Phys 52 : 137-143, 2002

17. Lingos TI, Recht A, Vicini F, Abner A, Silver $B$, Harris JR : Radiation pneumonitis in breast cancer patients treated with conservative surgery and radiation therapy. Int J Radiat Oncol Biol Phys 21 : 355-360, 1991

18. Crestani B, Kambouchner M, Soler P, Crequit J, Brauner M, Battesti JP, Valeyre D : Migratory bronchiolitis obliterans organizing pneumonia after unilateral radiation therapy for breast carcinoma. Eur Respir J 8 : 318-321, 1995

19. Prakash UB : Radiation-induced injury in the "nonirradiated" lung. Eur Respir J 13 : 715-717, 
1999

20. Ogo E, Komaki R, Fujimoto K, Uchida M, Abe T, Nakamura K, Mitsumori M, Sekiguchi K, Kaneyasu Y, Hayabuchi N : A survey of radiation- induced bronchiolitis obliterans organizing pneumonia syndrome after breast-conserving therapy in Japan. Int J Radiat Oncol Biol Phys $71: 123-131,2008$

21. Dorr W, Bertmann S, Herrmann T : Radiation induced lung reactions in breast cancer therapy. Modulating factors and consequential effects. Strahlenther Onkol 181:567-573, 2005

22. Koc M, Polat P, Suma S : Effects of tamoxifen on pulmonary fibrosis after cobalt-60 radiotherapy in breast cancer patients. Radiother Oncol $64: 171-175,2002$

23. Lind PA, Wennberg B, Gagliardi G, Rosfors S, Blom-Goldman U, Lidestahl A, Svane G : ROC curves and evaluation of radiation-induced pulmonary toxicity in breast cancer. Int $\mathrm{J}$ Radiat Oncol Biol Phys 64 : 765-770, 2006

24. Gagliardi G, Bjohle J, Lax I, Ottolenghi A, Eriksson F, Liedberg A, Lind P, Rutqvist LE: Radiation pneumonitis after breast cancer irradiation : analysis of the complication probability using the relative seriality model. Int J Radiat Oncol Biol Phys 46 : 373-381, 2000
25. Theuws JC, Kwa SL, Wagenaar AC, Boersma LJ, Damen EM, Muller SH, Baas P, Lebesque JV : Dose-effect relations for early local pulmonary injury after irradiation for malignant lymphoma and breast cancer. Radiother Oncol 48 : 33-43, 1998

26. Van Haecke P, Vansteenkiste J, Paridaens R, Van der Schueren E, Demedts M : Chronic lymphocytic alveolitis with migrating pulmonary infiltrates after localized chest wall irradiation. Acta Clin Belg 53 : 39-43, 1998

27. Taghian AG, Assaad SI, Niemierko A, Floyd $\mathrm{SR}$, Powell $\mathrm{SN}$ : Is a reduction in radiation lung volume and dose necessary with paclitaxel chemotherapy for node-positive breast cancer? Int J Radiat Oncol Biol Phys 62 : 386-391, 2005

28. Huang EY, Wang CJ, Chen HC, Sun LM, Fang FM, Yeh SA, Hsu HC, Hsiung CY, Wu JM : Multivariate analysis of pulmonary fibrosis after electron beam irradiation for postmastectomy chest wall and regional lymphatics : evidence for non-dosimetric factors. Radiother Oncol 57 : 91-96, 2000

29. Bentzen SM, Skoczylas JZ, Overgaard M, Overgaard J : Radiotherapy-related lung fibrosis enhanced by tamoxifen. J Natl Cancer Inst 88 : 918-922, 1996 